\title{
Is Platelet Rich Plasma Enriched Hydroxyapatite more Efficient than Hydroxyapatite alone in Extracted Third Molar Sockets? - A Study
}

\begin{abstract}
Ankit Singla ${ }^{1}$, Nishant Singh ${ }^{2}$, Shivendra Singh ${ }^{3}$, Meghna Kumar $^{4}$, Tanushree Debnath $^{5}$, Rahul Srivastava ${ }^{6}$
${ }^{1}$ Post Graduate Student, Department of Oral \& Maxillofacial Surgery, Rama Dental College-Hospital \& Research Centre, ${ }^{2}$ Professor, Department of Oral \& Maxillofacial Surgery, Rama Dental College-Hospital \& Research Centre, ${ }^{3}$ Assistant Professor, Department of Oral \& Maxillofacial Surgery, Rama Dental College-Hospital \& Research Centre, ${ }^{4}$ Post Graduate Student, Department of Oral \& Maxillofacial Surgery, Rama Dental College-Hospital \& Research Centre, ${ }^{5}$ Post Graduate Student, Department of Oral \& Maxillofacial Surgery, Rama Dental College-Hospital \& Research Centre, ${ }^{6}$ Reader, Department of Oral Medicine \& Radiology, Rama Dental College-Hospital \& Research Centre, India
\end{abstract}

Corresponding author: Dr. Shivendra Singh, Assistant Professor, Department of Oral \& Maxillofacial Surgery, Rama Dental College-Hospital \& Research Centre, India

DOI: http://dx.doi.org/10.21276/ijcmsr.2020.5.1.14

(c) BY-NC-ND

How to cite this article: Ankit Singla, Nishant Singh, Shivendra Singh, Meghna Kumar, Tanushree Debnath, Rahul Srivastava. Is platelet rich plasma enriched hydroxyapatite more efficient than hydroxyapatite alone in extracted third molar sockets? - a study. International Journal of Contemporary Medicine Surgery and Radiology. 2020;5(1):A59-A64.

\section{A B S T R A C T}

Introduction: Platelet-Rich Plasma (PRP) is defined as a concentrate of platelets, growth factors and cytokines, therefore it also promotes healing of bone and soft tissues. Hydroxyapatite is the major mineral component in bone, and synthetic apatites have become a common osteoconductive replacement material also, because of the internal architectural design of the scaffolds, for bone defects. This study is done to observe the efficacy of Platelet Rich Plasma in regeneration of bone when mixed with Hydroxyapatite (HA) and grafted in Mandibular third molar socket.

Materials and methods: A comparative non-randomized study was conducted, 10 patients who fulfilled the requirements were included in the study. The collected autologous PRP is mixed with hydroxyapatite graft dispensed into a sterile container and to form slurry. Thrombin plus Calcium chloride mixture is added to the slurry and waited for 2 min. A thick bolus of HA + PRP formed is now carried to the extracted left mandibular third molar socket. The contralateral mandibular third molar socket was filled with plain hydroxyapatite granules. A single operator carried out the procedure of Transalveolar removal of bilateral mandibular third molar under proper aseptic conditions. Post-operative radiographs (orthopantomograph) were recorded immediately after wound closure, and subsequently at the end of 1st month, 2nd month and 3rd month.

Results: Post-operative Trabecular pattern at the HA site and PRP mixed with HA site were analyzed at different time intervals using repeated measures and analysis of variance. Our observations were that the combination of PRP with bovine bone mineral (HA) was proved to be superior when observed with HA alone. During placement of graft into socket the mixture exhibited better handling properties.

Conclusion: Addition of PRP in hydroxyapatite bone graft provides a faster and better consolidation of the graft material and better osseointegration in the extraction sockets.

Keywords: PRP, HA, PRF, Osteoconductive

\section{INTRODUCTION}

Various graft materials are used nowadays for the purpose of reducing the signs and symptoms of post-operative period after surgical extraction and promotes bone regeneration and soft tissue healing. Among the various materials used to repair bone defects, autologous bone grafts are still considered as the gold standard. ${ }^{1-3}$ However, the use of platelet rich plasma (PRP) and Platelet rich fibrin (PRF) as a bone graft material has increased nowadays. The advantages rendered by growth factors from platelets were first described by Ross et al. ${ }^{4}$ Platelets trapped within fibrin matrix release certain growth factors which after activation stimulates the mitogenic response in the periosteum for bone repair. ${ }^{5}$ PRP contains various growth promoting factors such as platelet derived growth factors (PDGF), transforming growth factors (TGF), vascular endothelial growth factor (VEGF), and epithelial growth factor (EGF) that are responsible for increased collagen production, cell mitosis and recruitment of other cells to the site of injury, thereby initiating vascular ingrowths and cell differentiation (Table 1). PRP also contains certain cell adhesives like fibrin, fibronectin, and vitronectin responsible for osteoconduction and used as a matrix for bone, connective tissue, and epithelial migration. ${ }^{6}$ 
Bone is often subjected to various damages leading to its repair or regeneration ${ }^{7}$ which restores the bone to its original form and function. In the case of extraction socket healing, there is resorption of alveolar bone leading to an alteration of ridge contour and decrease in ridge volume which consequently impairs prosthetic rehabilitation. ${ }^{8}$ Special attention should be given for healing of bone following third molar extraction as it is associated with periodontal defects on the distal surface of an adjacent second molar. ${ }^{9}$

Porous hydroxyapatite (HA) material has been used to fill the bone defects, which has resulted in clinically acceptable responses. ${ }^{10}$ It has been shown that porous hydroxyapatite has an excellent bone conductive property which allows the growth of osteogenic cells from existing bone surfaces into adjacent bone graft material. ${ }^{11}$ Recently the combination of PRP with porous hydroxyapatite has been of great interest and has shown better healing as compared to porous hydroxyapatite used with saline alone. ${ }^{12}$ Studies have shown that hydroxyapatite is well tolerated by the surrounding tissues with no evidence of inflammation and is appropriate for application in humans. ${ }^{13,14}$ The purpose of this clinical study was to evaluate the osseous regeneration, clinically \& radiographically, after surgical removal of mandibular third molars with combination of PRP and porous hydroxyapatite.

\section{MATERIAL AND METHODS}

The present study has been undertaken on an outpatient basis in the Department of Oral and Maxillofacial surgery in Rama Dental College-Hospital \& Research Centre, Kanpur. Ten patients belonging to age group ranging from 20-40 years, with bilateral impacted mandibular third molars, were included in the study. Informed and a written consent was taken from the subjects prior to treatment. Ethical clearance for this study was granted by the Ethical committee of our institution.

\section{Inclusion criteria}

- Patients with bilateral unerupted mandibular third molar requiring similar surgical methods for their removal.

- Patients free of significant systemic diseases.

- Patients between 20 - 40 years of age.

\section{Exclusion criteria}

- Patients not giving consent for treatment.

- Patients undergoing bisphosphonate therapy.

\section{Graft material}

The graft material used in this study was Sybograf-Plus ${ }^{\mathrm{TM}}$ (Eucare Pharamaceuticals Pvt. Ltd.), a bioceramic composite material which contains $90 \%$ hydroxyapatite and $10 \%$ betatricalcium phosphate. This material is available in three forms i.e. blocks, cylinder and granules. In our present study, granules were used. These granules are white in colour measuring $600-700 \mu$ in size. The material is dispensed in a sterile vial of weight $0.5 \mathrm{~g}$ (fig-1).

\section{Preparation of PRP}

The platelet rich plasma was prepared by collecting peripheral blood from the ulnar vein of the patient in accordance with the procedures in force using single-use closed vacuum systems and glass tubes with sodium citrate as an anticoagulant. This is followed by two centrifugation steps. The first centrifugation step separates the red and white blood cells from plasma and platelets. The second centrifugation step further concentrates the platelets, producing the PRP separate from platelet-poor plasma. ${ }^{14}$ About $10-15$ min prior to use of the PRP in the surgical procedure, a coagulated gel was prepared by mixing PRP with $10 \%$ calcium chloride in a ratio of $10: 1$, i.e. $0.1 \mathrm{ml}$ of calcium chloride for every $1 \mathrm{ml}$ of PRP (fig-2).

\section{Operative technique}

In this study, bilateral impacted teeth were removed at same operating day. Under all aseptic conditions and local anesthesia impacted third molars were removed by a single operator only. After the removal of both teeth, HA enriched with PRP gel was placed in one socket and HA granules alone in another socket, which was selected randomly. The wound was closed primarily with 3-0 silk in simple interrupted fashion (fig-3,4,5).

\section{Follow-up}

Patients were recalled on first post-operative day, third day, seventh day, 8 weeks, 12 weeks, and 16 weeks postoperatively for follow-up study. The pain was evaluated at follow-up periods by using the visual analogue scale (VAS).${ }^{15}$ Evaluation of soft tissue healing was done by healing index healing index given by Landry et al. ${ }^{16}$ The scores were given on the basis of bleeding on palpation, epithelialization of margins, tissue color and presence or absence of suppuration. ${ }^{17}$

Intraoral periapical radiographs were taken and digitized using the standardized technique as advocated by Peretz et al. ${ }^{17}$ Radiographs were obtained at baseline, and at 8 th week, 12th week, 16th weeks postoperatively to compare and assess gray level histogram between PRP sites and HA site. X-ray machine was used at $65-70 \mathrm{kVp}$ and $10 \mathrm{~mA}$. These radiographs were placed on a light viewing box and digitalized using Canon EOS $1500 \mathrm{D}$ camera (ISO 1006400). The images were taken at same radiograph camera distance with a camera holder jig. The gray level histograms were obtained with the help of Image J (National institute of Health) software. ${ }^{19,20}$

\section{STATISTICAL ANALYSIS}

The three parameters i.e. Visual analogue scale, Soft tissue healing and bone density have been compared. The scores were analyzed by applying Mean, Standard Deviation, Variance, Range, Minimum and Maximum with Wilcoxon Signed Rank Test which compares the median values between the two groups. This test is non-parametric which is an alternative of Paired t-test. Statistical software i.e. Statistical Packages for Social Sciences (SPSS) 22.0 Version was used for the analysis of the data and Microsoft word and excel have been used to generate the graphs and tables.

\section{RESULTS}

There were eight male subjects and two female subjects who participated in the study. The subjects were in the age range from 18 years to 28 years, with a mean age of 22 years.

Assessment of pain by VAS: on the first day showed mean pain score of 1.5 in PRP + HA site and 2.6 in HA site, on the third day mean pain score was 1.0 in PRP + HA site and 2 in HA site, and on the seventh day score was 0 in both PRP 

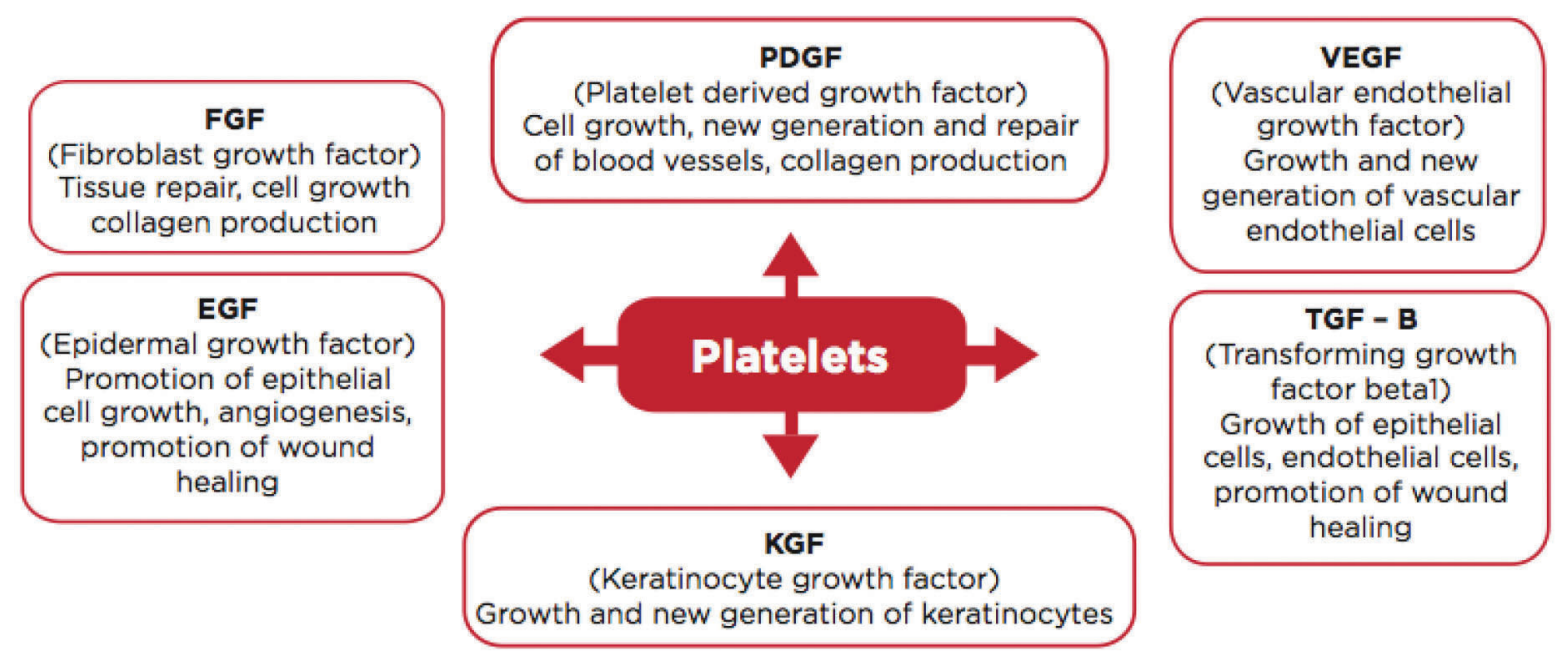

Table-1: Role of platelets and platelet derived growth factors
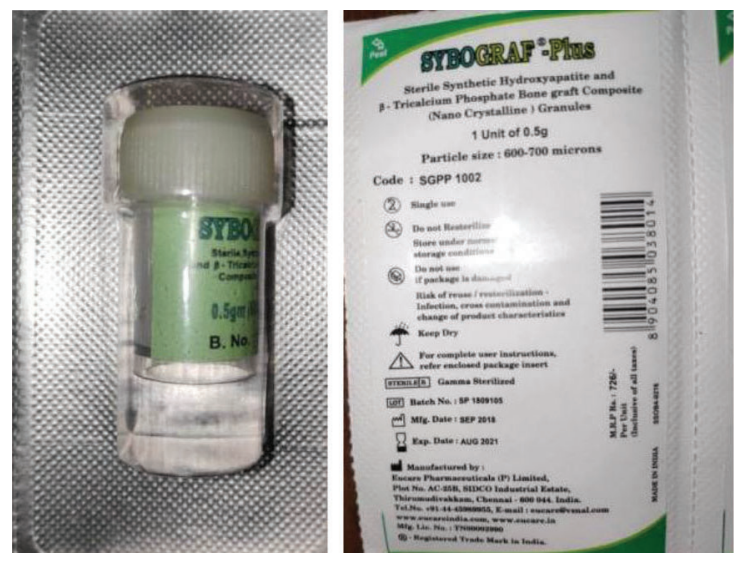

Figure-1: Sybograf- PlusTM bone graft material

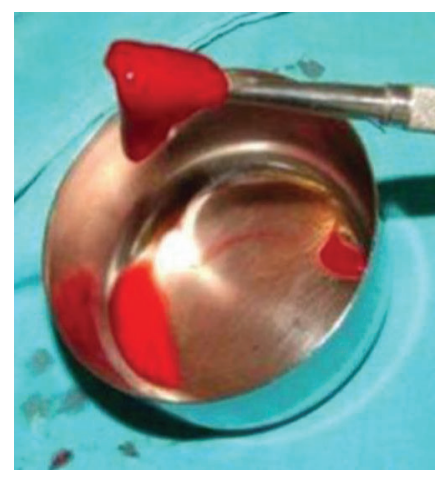

Figure-2: PRP gel after activation

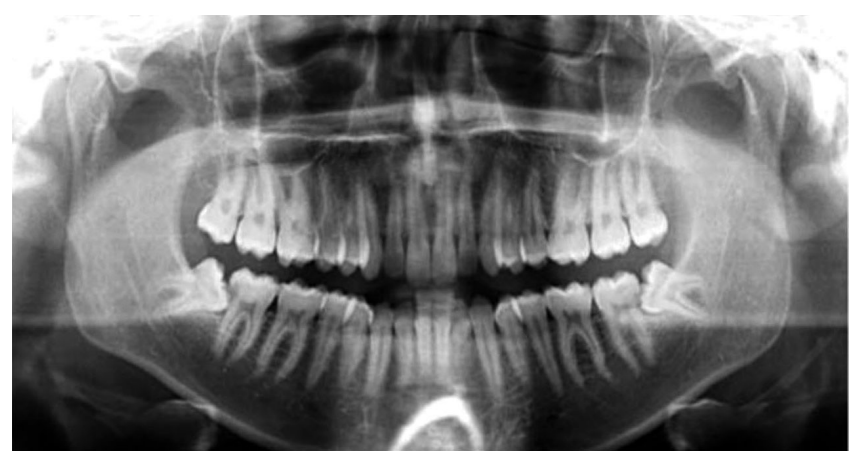

Figure-3: OPG showing bilaterally impacted mandibular third molars

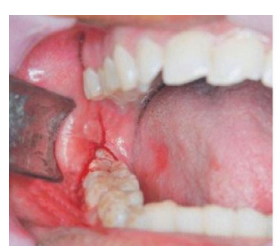

a)

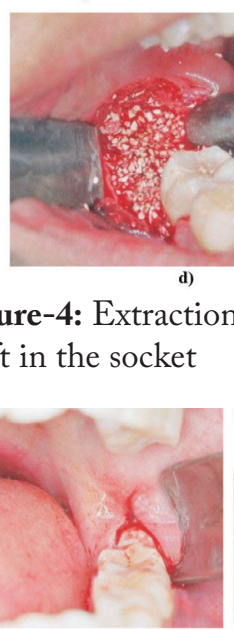

a)

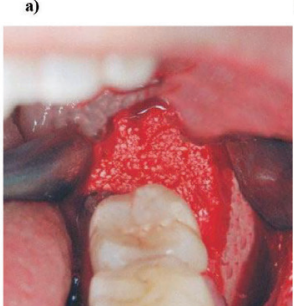

d)

b)
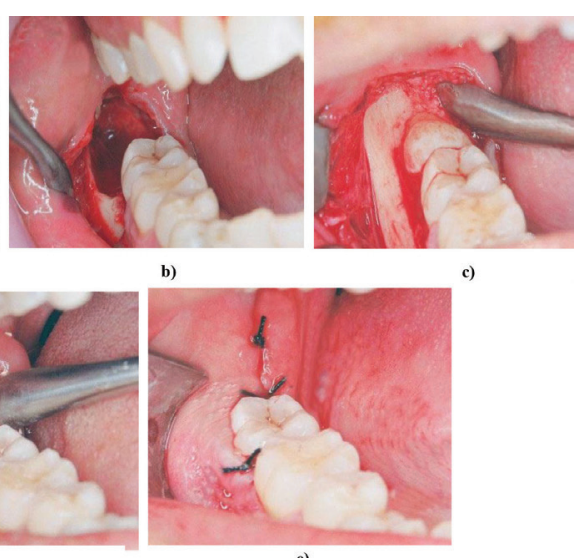

c)

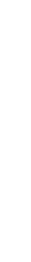

of HA
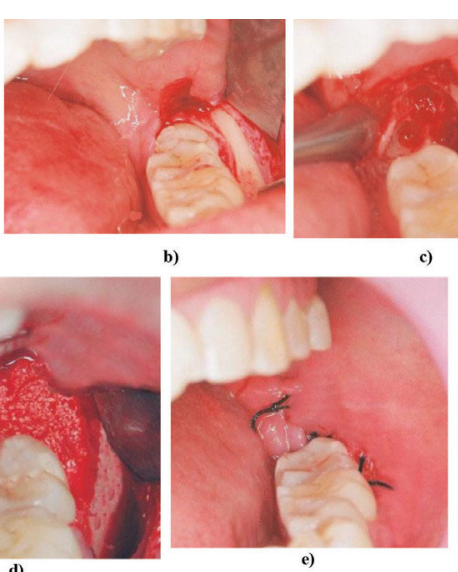

Fiagure-5: Extraction of third molar and placement of PRP enriched HA

+ HA and HA site. By doing the Mann-Whitney Test for comparison of $\mathrm{PRP}+\mathrm{HA}$ and $\mathrm{HA}$ it was found that there was a significant difference in pain on day 1 and day 3 with less pain in PRP + HA site [Fig.6].

Assessment of soft tissue healing by healing index: showed the mean score on the 1st day of 3.7 in PRP + HA site, 2.9 in $\mathrm{HA}$ site, on 3rd day 4.2 in PRP + HA site and 3.5 in $\mathrm{HA}$ site, on 7 th day mean score of 5.0 in PRP + HA site 


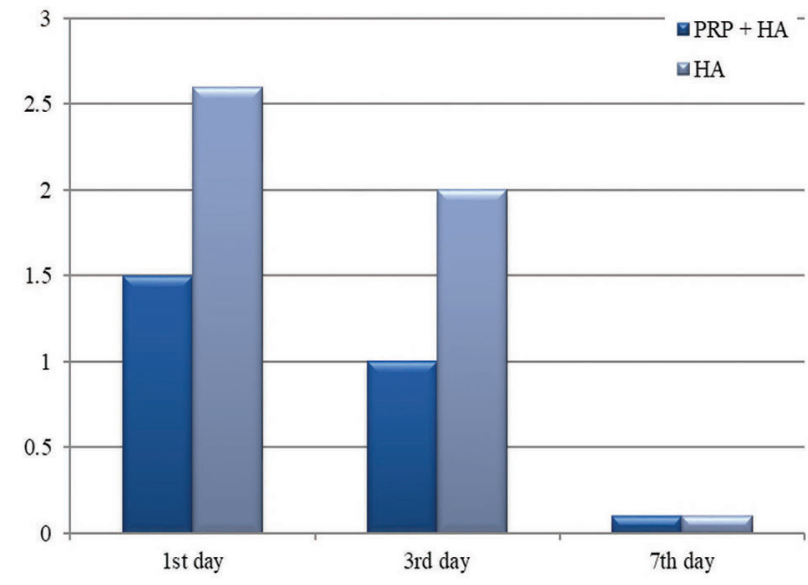

Figure-6: Difference in pain on day 1, day 3 and day 7

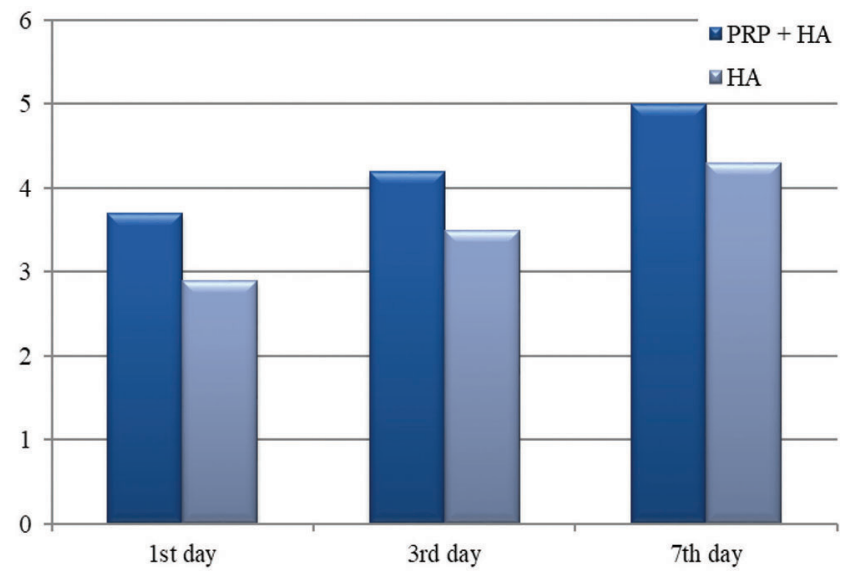

Figure-7: Difference in healing on day 1, day 3 and day 7

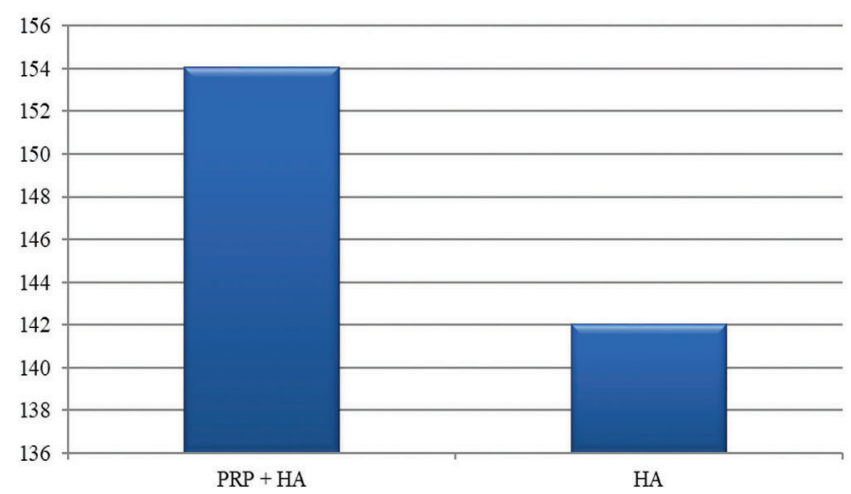

Figure-8: Radiological assessment of grey values at 4 months

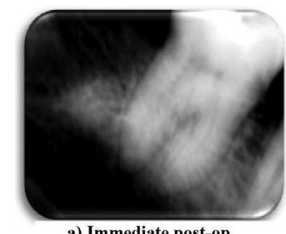

a) Immediate post-op

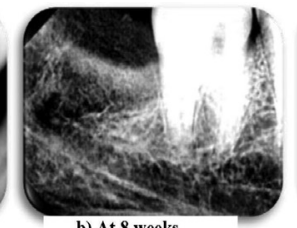

b) At 8 weeks

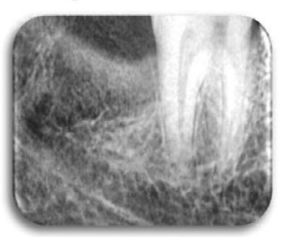

d) At 16 weeks

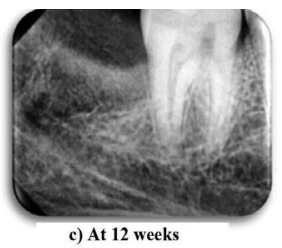

Figure-9: Extraction of third molar and placement of HA bone graft

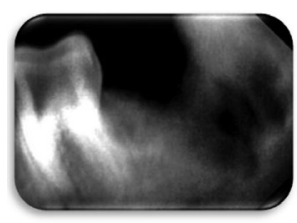

a) Immediate post-op

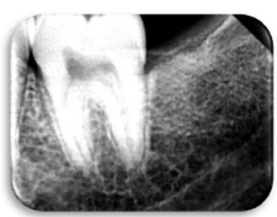

b) At 8 weeks

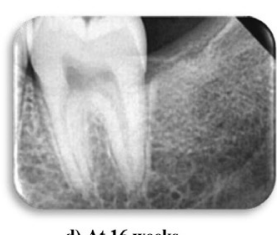

d) At 16 weeks

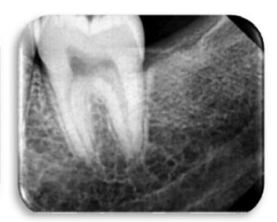

c) At 12 weeks
Figure-10: Extraction of third molar and placement of PRP enriched HA

and 4.3 in HA site. By doing the Mann-Whitney Test for comparison of PRP + HA and HA it was found that there was a significant difference in healing on day 1 and day 3 with better healing in PRP + HA site [Fig.7].

\section{Assessment of bone density:}

At 8 weeks: Blending of bone margins was seen in three patients in PRP + HA site and four patients in HA site. Chi-square test showed $p$ value of $\leq 0.001$. The formation of trabecular bone was seen in nine patients at PRP + HA site and nine patients at HA site also.

At 12 weeks: Blending of bone margins was seen in all the 10 patients in both PRP and HA sites and the formation of trabecular bone was seen in all 10 patients in PRP site and HA site.

At 16 weeks: Blending of bone was seen in all 10 patients in both PRP site and HA site. The formation of trabecular bone was seen in all 10 patients in both the sites. Assessment of gray level values at 16 weeks showed that the average gray scale value for PRP site was 145.29 and for HA site it was 139.04 [Fig-8].

Bone density can be measured by calculating the gray level value on the radiographs. All the radiographs were taken and digitalized using the standardized technique as stated by Peretz et al. ${ }^{18}$ Gray level values can be measured with the help of different softwares available such as Image J (National Institute of Health), and Adobe Photoshop (Adobe Systems). ${ }^{20,21}$ The radiological assessment in the follow-up period of 4 months showed radiological evidence of osseous in-growths into both the extraction socket defect. The evaluation of bone density was done by Image J software. The radiological assessment showed the grey level values calculated at 4 months at the PRP site 154.8 were comparatively higher than the HA site 141.06 (fig-9,10).

\section{DISCUSSION}

Use of PRP is an innovative technique to improve bone healing. A number of studies have been done to check the efficacy of PRP with different bone substitutes. Various studies have verified that PRP when combined with autogenous bone results in considerably faster radiographic maturation and histomorphometrically denser bone regeneration. ${ }^{22}$ But they have their own disadvantages like, donor site morbidity, limited availability, post-operative discomfort for the patient 
and increased surgical time. On the other hand,varying results of success have been demonstrated when PRP is added to allografts. But there is a risk of transmission of $\mathrm{HIV}$ infection occurring from banked bone. Thus the use of other bone substitutes like alloplastic bone substitute is recommended. Hydroxyapatite (HA) is an osteoconductive material which is biologically inert, readily available, and easily adaptable to the site in terms of size and shape. Hydroxyapatite does not exhibit osteoinductive capacity but does demonstrate significant osteoconductive potential. ${ }^{23} \mathrm{PRP}$ and HA offers an interesting and clinically useful modality to the clinician in treating certain periodontal osseous defects. ${ }^{24}$ The present study was undertaken to study the efficacy of platelet-rich plasma in regeneration of bone when mixed with porous hydroxyapatite and grafted in mandibular third molar socket. This was in accordance with the study by Dr. Kazuhiro Okuda (2005). ${ }^{24}$ The bone formation was measured at different intervals post-operatively. It was observed after a time interval of 3 months that, the sockets filled with the combination of graft and activated platelet rich plasma, showed increase in bone formation, compared to sockets which were left as such and the results were confirmed radiographically. This was in accordance with the study by R.E. Marx (1998). ${ }^{25}$

In the present study a clinical trial was carried out to compare the effectiveness of PRP and synthetic granules of hydroxyapatite in terms of better bone regeneration and soft tissue healing. Various factors affect bone healing that can vary from case to case, and so to avoid such bias both the materials were placed in different extraction sockets of the same individual in the same sitting. Soft tissue healing was evaluated using gingival healing index by Landry et al., which showed better soft tissue healing of extraction sockets with PRP as compared to HA alone sockets. This finding is supported by the authors who in their study reported that there was a decreased rate of alveolar osteitis, objectively faster soft tissue flap healing and decreased swelling in the extraction sockets treated with PRP.

Bone density is measured by calculating the gray level value on the radiographs. All the radiographs were taken and digitalized using the standardized technique as stated by Peretz et al..$^{25}$ Gray level values can be measured with the help of different softwares such as Image J (National Institute of Health), and Adobe Photoshop (Adobe Systems).The radiological assessment in the follow-up period of 4 months showed radiological evidence of osseous in-growths into both the extraction socket defect.

\section{CONCLUSION}

Our observations in the present study indicate that Hydroxyapatite could be successfully used with or without PRP for socket grafting in dentistry. But a faster and better consolidation of graft material and a better osseointegration can be understood with the addition of PRP in the Hydroxyapatite graft material. Growth factors in general and PRP in particular are part of a new biotechnology with already established efficacy and future potentials. It is the responsibility of the clinician to gain a thorough understanding of this biotechnology and to use it correctly and wisely for the benefit of patients.

\section{REFERENCES}

1. Aghazadeh A, Rutger Persson G, Renvert S. A single centre randomized controlled clinical trial on the adjunct treatment of intra bony defects with autogenous bone or a xenograft: Results after 12 months. J Clin Periodontol 2012; 39(1):666-73.

2. Meyle J, Hoffmann T, Topoll H, Heinz B, Al Machot E, Jervoe Storm PM, et al. A multicentre randomized controlled clinical trial on the treatment of intrabony defects with enamel matrix derivatives/synthetic bone graft or enamel matrix derivatives alone: Results after 12 months. J Clin Periodontol 2011;38(2):652-60.

3. Misch CM. Autogenous bone: Is it still the gold standard? Implant Dent 2010; 19(3):361.

4. Ross R, Glomset J, Kariya B, Harker L. A platelet dependent serum factor that stimulates the proliferation of arterial smooth muscle cells in vitro. Proc Natl Acad Sci U S A 1974; 71(5):1207-10.

5. Gassling V, Douglas T, Warnke PH, Açil Y, Wiltfang J, Becker ST. Platelet rich fibrin membranes as scaffolds for periosteal tissue engineering. Clin Oral Implants Res 2010; 21(2):543-9.

6. Marx RE. Platelet rich plasma: Evidence to support its use. J Oral Maxillofac Surg 2004; 62(4):489-96.

7. Intini $G$. The use of platelet rich plasma in bone reconstruction therapy. Biomaterials 2009;30(6):495666.

8. Artzi Z, Tal H, Dayan D. Porous bovine bone mineral in healing of human extraction sockets. Part 1: Histomorphometric evaluations at 9 months. J Periodontol 2000; 71(5):1015-23.

9. Kugelberg CF, Ahlström U, Ericson S, Hugoson A, Kvint S. Periodontal healing after impacted lower third molar surgery in adolescents and adults. A prospective study. Int J Oral Maxillofac Surg 1991; 20(3):18-24.

10. Kenney EB, Lekovic V, Han T, Carranza FA Jr, Dimitrijevic B. The use of a porous hydroxyapatite implant in periodontal defects. Clinical results after six months. J Periodontal 1985;56(1):82-88

11. Holmes RE, Wardrop RW. Hydroxyapatite as a bone graft substitute in orthognathic surgery: histologic and histometric findings. J Oral Maxillofac Surg 1998;46(6):661-671

12. Gonshor A.Technique for producing platelet rich plasma and platelet concentrate: background and process. Int J Periodontics Restorative Dent 2002;22(4):547-557

13. Okuda K, Tai H, Tanabe K, Suzuki H, Sato T, Kawase T, Saito Y, Walff L, Yoashie H. Platelet rich plasma combined with porous hydroxyapatite graft for the treatment of intrabony periodontal defects in humans. A comparative controlled clinical study. J Periodontal 2005;76(2):890-898

14. Lykins CL, Friedman CD, Costantino PD. Hydroxyapatite cement in craniofacial skeletal reconstruction and its effects on the developing craniofacial skeleton. Arch Otolaryngol Head Neck Surg 1998;124(1):153-159.

15. Isik K, Unsal A, Kalayci A, Durmus E. Comparison of three pain scales after impacted third molar surgery. Oral Surg Oral Med Oral Pathol Oral Radiol Endod 2011; 112(6):715-8. 
16. Landry RG, Turnbull RS, Howley T. Effectiveness of benzydamyne $\mathrm{HCl}$ in the treatment of periodontal postsurgical patients. Res Clin Forum 1988; 10(3):10518.

17. Jankovic SM, Zoran AM, Lekovic MC, Bozidar DS, Kenneyy BE. The use of platelet Rich plasma in combination with connective tissue grafts following treatment of gingival recessions. Periodontal Pract Today 2007; 4(5):63-71.

18. Peretz B, Kaffe I, Amir E. Digital images obtained with a digital camera are not associated with a loss of critical information - A preliminary study. Br Dent J 2009; 206(2):E9.

19. Goes P, Lima AP, Melo IM, Rêgo RO, and Lima V. Effect of atorvastatin in radiographic density on alveolar bone loss in wistar rats. Braz Dent J 2010; 21(3):193-8.

20. Grandi T, Garuti G, Guazzi P, Sapio U, Forabosco A. A longitudinal, Multi-centre study on the relationship between insertion torque and peri-implant bone resorption. J Clin Pract Oral Implantol 2010; 1(4):3340.

21. Damante JH, Da S Guerra EN, Ferreira O Jr. Spontaneous resolution of simple bone cysts. Dentomaxillofac Radiol 2002; 31(1):182-6.

22. Marx R, Garg A Dental and craniofacial applications of platelet rich plasma. Quintessence 1. edition 2005.

23. Okuda K, Tai H, Tanabe K, Suzuki H, Sato T, Kawase T, Saito Y, Walff L, Yoashie H. Platelet rich plasma combined with porous hydroxyapatite graft for the treatment of intrabony periodontal defects in humans. A comparative controlled clinical study. J Periodontal 2005;76(5):890-898.

24. Lykins CL, Friedman CD, Costantino PD. Hydroxyapatite cement in craniofacial skeletal reconstruction and its effects on the developing craniofacial skeleton. Arch Otolaryngol Head Neck Surg 1998;124(4):153-159.

25. Marx RE, Carlson ER, Eichstaedt RM, Schimmele SR, Strauss JE, Geoegeff KR. Platelet rich plasma growth factor enhancement for bone grafts. Oral Surg Oral Med Oral Pathol Oral Radiol Endod 1998;85(3):638646.

26. Marx RE. Platelet rich plasma: evidence to support its use. J Oral Maxillofac Surg 2004;62(5):489-496.

27. Hanna R, Trejo PM, Weltman RL. Treatment of intrabony defects with bovine-derived xenograft alone and in combination with platelet rich plasma: a randomized clinical trial. J Periodontol 2004;75(1):1668-1677.

Source of Support: Nil; Conflict of Interest: None

Submitted: 02-12-2019; Accepted: 30-12-2019; Published online: 24-01-2020 